\title{
Dysregulation of PI3K and Hippo signaling pathways synergistically induces chronic pancreatitis via CTGF upregulation
}

\author{
Takeshi Tamura, ${ }^{1}$ Takahiro Kodama, ${ }^{1}$ Katsuhiko Sato, ${ }^{1}$ Kazuhiro Murai, ${ }^{1}$ Teppei Yoshioka, ${ }^{1}$ Minoru Shigekawa, ${ }^{1}$ Ryoko Yamada, ${ }^{1}$ \\ Hayato Hikita, ${ }^{1}$ Ryotaro Sakamori, ${ }^{1}$ Hirofumi Akita, ${ }^{2}$ Hidetoshi Eguchi, ${ }^{2}$ Randy L. Johnson, ${ }^{3}$ Hideki Yokoi, ${ }^{4}$ Masashi Mukoyama, ${ }^{5}$ \\ Tomohide Tatsumi, ${ }^{1}$ and Tetsuo Takehara' \\ 1Department of Gastroenterology and Hepatology and ${ }^{2}$ Department of Gastroenterological Surgery, Osaka University Graduate School of Medicine, Suita, Osaka, Japan. ${ }^{3}$ Department of Cancer Biology, The \\ University of Texas MD Anderson Cancer Center, Houston, Texas, USA. ${ }^{4}$ Department of Nephrology, Kyoto University Graduate School of Medicine, Kyoto, Japan. ${ }^{5}$ Department of Nephrology, Kumamoto \\ University Graduate School of Medical Sciences, Kumamoto, Japan.
}

\begin{abstract}
The role of PI3K and Hippo signaling in chronic pancreatitis (CP) pathogenesis is unclear. Therefore, we assessed the involvement of these pathways in CP by examining the PI3K and Hippo signaling components PTEN and SAV1, respectively. We observed significant decreases in pancreatic PTEN and SAV1 levels in 2 murine CP models: repeated cerulein injection and pancreatic ductal ligation. Additionally, pancreas-specific deletion of Pten and Sav1 (DKO) induced CP in mice. Pancreatic connective tissue growth factor (CTCF) was markedly upregulated in both CP models and DKO mice, and pancreatic CCAAT/ enhancer-binding protein- $\alpha$ (CEBPA) expression was downregulated in the CP models. Interestingly, in pancreatic acinar cells (PACs), CEBPA knockdown reduced PTEN and SAV1 and increased CTCF levels in vitro. Furthermore, CEBPA knockdown in PACs induced acinar-to-ductal metaplasia and activation of cocultured macrophages and pancreatic stellate cells. These results were mitigated by CTCF inhibition. CP in DKO mice was also ameliorated by Ctg $f$ gene deletion, and cerulein-induced CP was alleviated by antibody-mediated CTCF neutralization. Finally, we observed significantly decreased PTEN, SAV1, and CEBPA and increased CTCF levels in human CP tissues compared with nonpancreatitis tissues. Taken together, our results indicate that dysregulation of PI3K and Hippo signaling induces CP via CTCF upregulation.
\end{abstract}

\section{Introduction}

Chronic pancreatitis (CP) is characterized by continuous inflammatory destruction of the pancreas and replacement with fibrotic tissues, leading to the permanent loss of pancreatic function (1). CP is caused by heavy alcohol consumption and genetic mutations related to pancreatic digestive enzymes such as cationic trypsinogen (PRSS1), serine protease inhibitor Kazal type 1 (SPINK1), and carboxypeptidase A1 (CPA1) (2). However, $10 \%-30 \%$ of CP cases do not have identifiable causative factors, suggesting the existence of unknown etiological factors (3). Although premature intra-acinar trypsinogen activation is a key initiating factor of pancreatitis, the pathogenesis of CP is not fully elucidated, and no effective or specific treatment exists. Therefore, further understanding of this pathogenic mechanism at the cellular and molecular levels is important to identify new therapies to prevent CP progression.

During continuous pancreatic damage, acinar cells transdifferentiate into a progenitor-like cell type with ductal character-

Authorship note: T. Tamura and T. Kodama are co-first authors.

Conflict of interest: The authors have declared that no conflict of interest exists.

Copyright: () 2021, American Society for Clinical Investigation.

Submitted: August 18, 2020; Accepted: May 11, 2021; Published: July 1, 2021

Reference information: J Clin Invest. 2021;131(13):e143414

https://doi.org/10.1172/JCl143414. istics. This process is called acinar-to-ductal metaplasia (ADM), and its appearance is an important morphological characteristic of CP (4). Several animal experiments have shown that ADM can develop into pancreatic intraepithelial neoplasia (PanIN) and subsequently into pancreatic ductal adenocarcinoma (PDAC) (5). Indeed, CP is recognized to be one of the strongest risk factors for human PDAC (6). Several signaling pathways, including the PI3K $(7,8)$ and Hippo signaling pathways (9), are reported to be involved in this oncogenic process. The PI3K pathway is an oncogenic signaling pathway that promotes cell proliferation and differentiation (10). Animal studies have shown that haploinsufficiency of Pten, which is a negative regulator of the PI3K signaling pathway, promotes PDAC development and progression in Kras-mutant mice $(7,8)$. Furthermore, PTEN (phosphatase and tensin homolog) expression has been reported to be downregulated in most human PDACs (8), indicating that PI3K pathway activation is the driving force of PDAC development. The Hippo signaling pathway controls organ size in animals by restricting cell proliferation and promoting apoptosis, both of which are also important for tumor suppression (11). Its main component is a kinase cascade wherein mammalian STE20-like protein kinase 1 (MST1) and MST2, in complex with Salvador homolog 1 (SAV1), phosphorylate and activate the large tumor suppressor 1 (LATS1) and LATS2 kinases, which in turn phosphorylate and inactivate the Hippo effector YES-associated protein (YAP). Hippo signal- 
ing inactivation, as represented by SAV1 downregulation or YAP activation, is correlated with poor overall survival in patients with PDAC (12-14), suggesting tumor-suppressive roles of the Hippo pathway in PDAC.

Moreover, dysregulation of the PI3K and Hippo signaling pathways has been observed in human CP $(15,16)$. More than half of CP patients exhibit phosphorylation of RPS6, a major downstream effector of the PI3K/AKT/mTOR pathway, in the pancreas, suggesting activation of PI3K signaling. In addition, upregulation of YAP expression was observed in pancreatic tissue in a murine model of pancreatitis and human patients with $\mathrm{CP}$, suggesting inactivation of Hippo signaling $(9,15)$. However, the pathological significance of these dysregulated signaling pathways in $\mathrm{CP}$, and their regulatory mechanisms, are not well understood. In the present study, we found that downregulation of PTEN and SAV1 contributes to dysregulation of the PI3K and Hippo signaling pathways in murine CP models and human CP patients. We showed that mice with genetic disruption of Pten and Sav1 spontaneously develop severe $\mathrm{CP}$, demonstrating the importance of these signaling pathways in CP development. We also identified CCAAT/ enhancer-binding protein- $\alpha$ (CEBPA) as the upstream regulator of both PTEN and SAV1 and showed that inactivation of CEBPA in pancreatic acinar cells (PACs) induces ADM and the activation of macrophages and pancreatic stellate cells (PSCs) via upregulation of connective tissue growth factor (CTGF). Finally, we showed that CTGF inhibition markedly ameliorates CP induced by either deletion of Pten/Sav1 or repeated injection of cerulein in mice, suggesting CTGF as a novel therapeutic target in CP.

\section{Results}

The expression of PTEN and SAV1 is downregulated in the pancreatic tissues of mice in 2 models of CP. To clarify the roles of the PI3K and Hippo signaling pathways in CP pathogenesis, we used 2 major murine models of CP: repeated administration of cerulein (17) and pancreatic duct ligation (18). The pancreas atrophied in both CP models (Supplemental Figure 1, A and B; supplemental material available online with this article; https://doi.org/10.1172/ JCI143414DS1), and histological examination showed a reduced number of acinar cells and the emergence of ductal structures in the pancreas (Figure 1, A and B). We performed immunohistochemical staining for the ADM marker SOX9 and found that the pancreata of CP model mice showed an increased number of SOX9-positive transdifferentiated ADM lesions in comparison with control mice (Figure 1, A and B). CP is also characterized by chronic inflammation and fibrogenesis, which are triggered by inflammatory macrophages and PSCs, respectively (17). Indeed, both CP models showed macrophage infiltration in the pancreas, as indicated by the significant increase in Cd68 expression, leading to marked production of inflammatory cytokines and chemokines, including Tnfa, Il1b, and Ccl2 (Figure 1, C and D). In addition, the expression levels of the profibrogenic $\mathrm{Tg} f b 1$ gene and type I collagen (Col1a1 and Col1a2) genes were significantly upregulated in the pancreas in both CP models, with widespread deposition of Sirius red-positive fibers, indicating activation of PSCs in these models (Figure 1, C and D). Next, we examined the expression levels of Pten and Sav1, major components of the PI3K and Hippo signaling pathways, respectively. Their mRNA levels were significantly lower in the pancreata of mice in both $\mathrm{CP}$ models than in those of control mice (Figure 1, E and F). Consistent with this finding, immunohistochemical staining for PTEN and SAV1 showed that their protein levels were also significantly lower in acinar cells of mice in both CP models than in those of control mice (Figure 1, G and H). Thus, AKT and YAP, which are the effector proteins of PI3K and Hippo signaling, respectively, were activated in the pancreas in both CP models, as demonstrated by the increase in activating phosphorylation of AKT and the decrease in inhibitory phosphorylation of YAP by Western blot analysis (Figure 1, I and J), along with the increase in phosphorylated AKT and nuclear YAP levels in acinar cells by immunohistochemistry (Supplemental Figure 1, C and D). We also found significant upregulation of Ctgf, CCN family proteins (19), and downstream transcriptional targets of the Hippo signaling pathway (20) in mice in both CP models compared with control mice (Figure 1K). Taken together, these results indicate that both the PI3K and Hippo signaling pathways are dysregulated in CP with the downregulation of PTEN and SAV1.

Mice with pancreas-specific loss of Pten and Sav1 spontaneously develop CP. To investigate the significance of PI3K and Hippo signaling pathway dysregulation in $\mathrm{CP}$, we generated mice with pancreas-specific Pten and/or Sav1 knockout (KO) (Supplemental Figure 2, A and B). Single knockout of either Pten or Sav1 in mice (Pdx1-Cre Pten ${ }^{f / f l}[\mathrm{PTEN}-\mathrm{KO}]$ and Pdx1-Cre Sav1 ${ }^{f / f l}$ [SAV1$\mathrm{KO}$ ] mice) did not affect pancreas weight or pancreatic histology at 6 weeks of age (Figure 2, A-C). In sharp contrast, the pancreata of mice with deletion of both Pten and Sav1 (Pdx1-Cre Pten ${ }^{f / f l}$ $S a v 1^{f / f l}$ [double-KO; DKO] mice) were extremely atrophied (Figure 2A) and weighed significantly less than those of control $\left(\right.$ Pten $^{f / f l}$ Sav1 $^{f / f l}$ [wild-type; WT] mice) and single-knockout mice (Figure 2B). Histological examination showed massive loss of acinar cells, disorganized pancreatic ductal hyperplasia, the emergence of structures reminiscent of SOX9-positive ADM lesions, and fibrosis in the pancreata of DKO mice (Figure 2C). These results indicated that mice with loss of both Pten and Sav1 spontaneously develop CP. However, the presence of glucagon-positive islet cells and the levels of serum glucose and insulin did not differ between DKO mice and the other strains of mice (Supplemental Figure 2, C and D), suggesting that pancreatic endocrine functions were maintained in DKO mice. When we aged the mice longer, PTEN-KO and SAV1-KO mice survived and developed a small amount of ADM in their pancreata at 10 months of age (Supplemental Figure 2E). Meanwhile, all DKO mice died by 8 weeks of age without developing PanIN or PDAC (Supplemental Figure 2F).

Next, we investigated the phenotypes of DKO mice at early ages after birth. The weight of the pancreas increased gradually in DKO mice and did not differ from that of other mice until 2 weeks of age (Supplemental Figure 2G), excluding the possibility of failed pancreatic development in DKO mice. From 2 weeks of age, the weight of the pancreas started to decrease over time, and pancreatic tissues were almost completely replaced by ADM and fibrotic lesions at 6 weeks of age (Supplemental Figure 2, H and I). As expected, an increase in activating phosphorylation of AKT and a decrease in inhibitory phosphorylation of YAP were observed in the pancreata of DKO mice (Figure 2D), suggesting activation of AKT and YAP. Given the stimulatory roles of AKT 
A

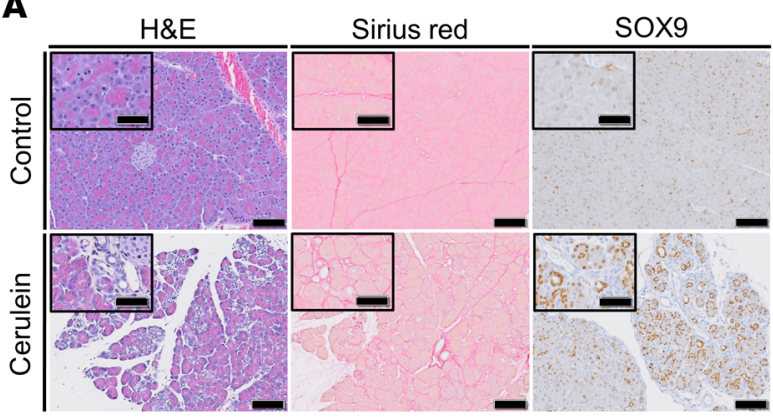

C

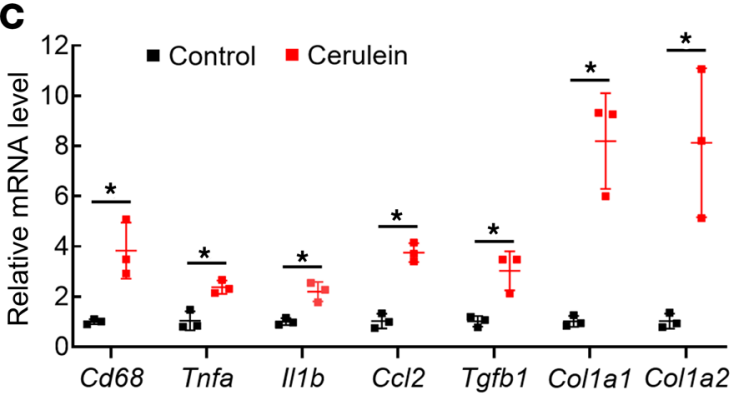

B

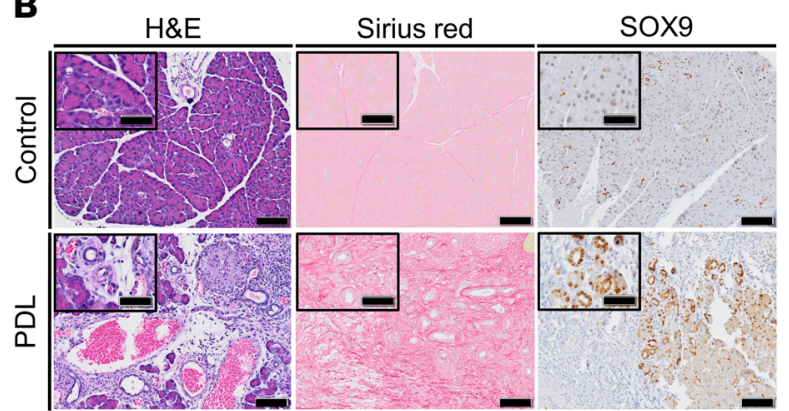

D

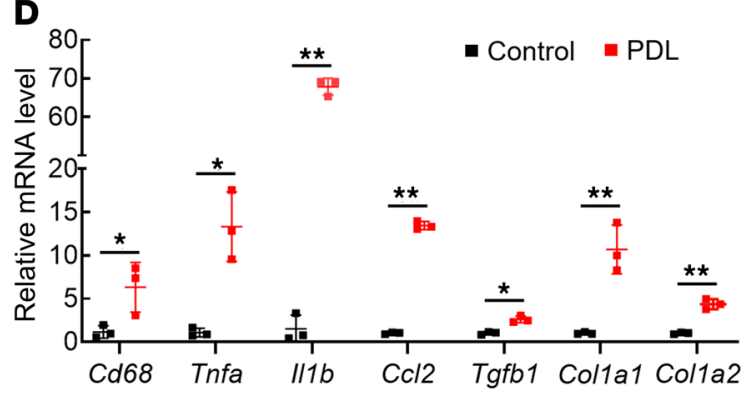

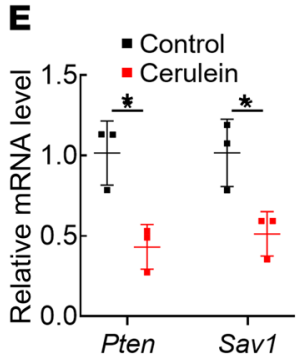

\section{$\mathbf{F}$}

ब 1.5 - PDL

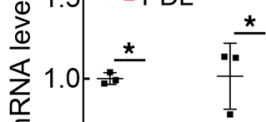

ह

$\stackrel{0}{\geq} 0.5$

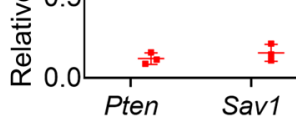

G
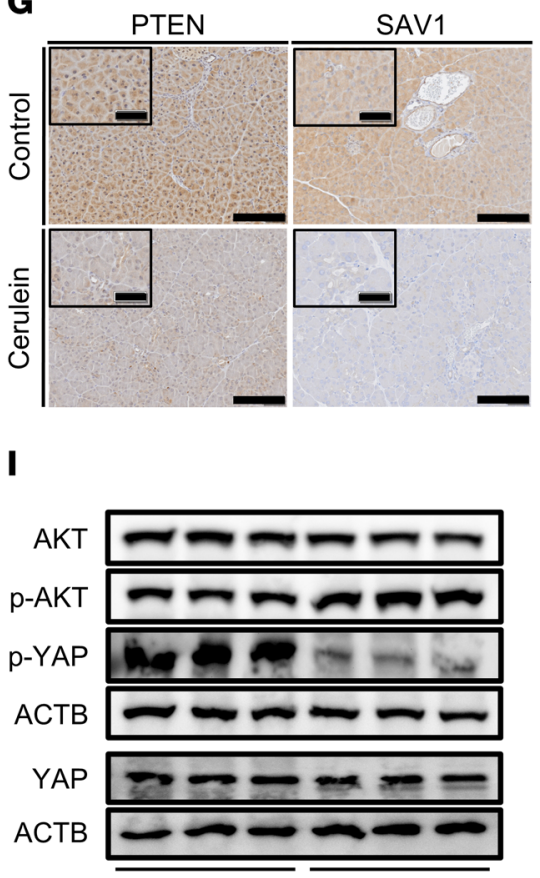

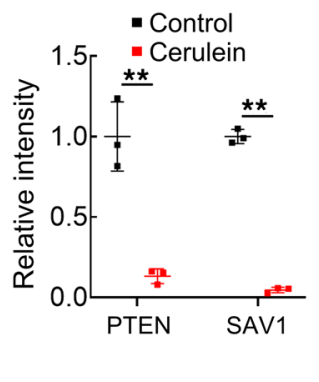

$\mathbf{J}$

H
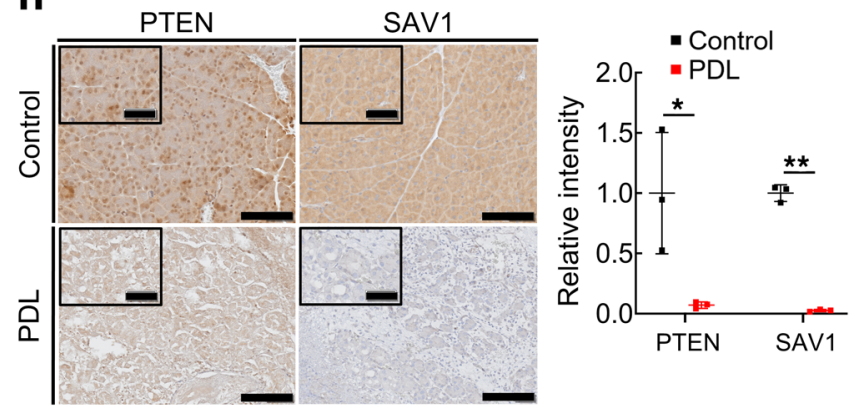

K

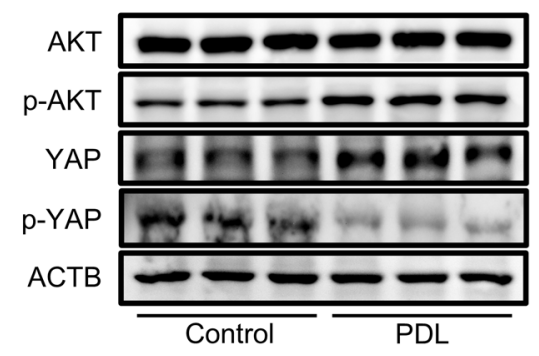


Figure 1. Expression of PTEN and SAV1 is downregulated in the pancreatic tissues of mice in $\mathbf{2}$ models of CP. (A and B) Representative images of H\&E, Sirius red, and SOX9 staining of pancreatic tissue in mice after repeated cerulein or vehicle (control) injection (A) and in mice subjected to pancreatic duct ligation (PDL) surgery or sham surgery (B). (C and D) Cd68, Tnfa, I/1b, Ccl2, Tgfb1, Col1a1, and Col1a2 mRNA levels in pancreatic tissue in mice after repeated cerulein injection (C) and in mice subjected to PDL surgery (D). (E and F) Pten and Sav1 mRNA levels in pancreatic tissue in mice after repeated cerulein injection (E) and in mice subjected to PDL surgery (F). (G and $\mathbf{H})$ Representative images of PTEN and SAV1 staining of pancreatic tissue in mice after repeated cerulein injection (G, left), with quantification of the PTEN and SAV1 staining intensity (G, right); and in mice subjected to PDL surgery ( $\mathbf{H}$, left), with quantification of PTEN and SAV1 staining intensity ( $\mathbf{H}$, right). (I and J) Protein levels of AKT, p-AKT, YAP, p-YAP, and ACTB in the pancreata of mice after repeated cerulein injection (I) and in mice subjected to PDL surgery (J). (K) Ctgf mRNA levels in pancreatic tissue in mice after repeated cerulein injection (left) and in mice subjected to PDL surgery (right). Blots run in parallel contemporaneously or run at different times with loading control for each gel are shown. All data are presented as the means \pm SDs of results for 3 mice per group. Student's $t$ test was used to evaluate differences between 2 groups. ${ }^{*} P<0.05$ and ${ }^{* *} P<0.005$. Scale bars: $100 \mu \mathrm{m}$ and $50 \mu \mathrm{m}$ (insets).

and YAP activation in cell proliferation, we assessed the number of Ki67-positive proliferating cells in the pancreas. While a mild increase in the number of Ki67-positive acinar cells was observed in PTEN-KO and SAV1-KO mice compared with WT mice, a massive increase in Ki67-positive ductal cells was seen in DKO mice (Supplemental Figure 2J). The number of apoptotic acinar cells in the pancreas, as determined by TUNEL staining, was increased in DKO mice compared with the other strains of mice (Supplemental Figure $2 \mathrm{~K}$ ). Taken together, these data suggest that apoptosis of acinar cells and proliferation of ductal cells may contribute to $\mathrm{CP}$ phenotypes in DKO mice. We then examined the expression levels of inflammatory cytokines/chemokines, fibrosis-related genes, and ADM-related genes in DKO mice. Similar to the pattern in both CP models, the mRNA levels of Cd68, Tnfa, Il1b, Ccl2, Tgfb1, Col1a1, Col1a2, and Ctgf were all significantly increased in the pancreata of DKO mice (Figure 2E), suggesting that pancreas-specific co-deletion of PTEN and SAV1 recapitulates the CP phenotypes observed in these 2 major CP models.

CEBPA positively regulates PTEN and SAV1 in acinar cells, and inhibition of PTEN and SAV1 induces ADM through CTGF upregulation in vitro. We then assessed the effects of PTEN and SAV1 loss in acinar cells in vitro. To this end, we knocked down PTEN and/or SAV1 in the mouse PAC tumor cell line 266-6 and found that the double knockdown of PTEN and SAV1 strongly upregulated the expression of SOX9, increased the expression of CTGF, and decreased the inhibitory phosphorylation of YAP (Figure 3A). These data suggest that the inhibition of PTEN and SAV1 induces $\mathrm{ADM}$ in vitro, recapitulating PAC phenotypes in both CP models and in DKO mice.

Next, we examined an upstream transcription factor (TF) that regulates Pten and Sav1 in the pancreas. In silico analysis using the TRANSFAC TF database (21) revealed that Cebpa was the curated TF that targeted Pten and Sav1. The pancreatic expression of Cebpa was significantly downregulated in mice in both models of experimental CP compared with control mice (Figure 3B).
We thus knocked down CEBPA in the mouse PAC tumor cell line 266-6 and found that CEBPA knockdown significantly decreased the mRNA and protein levels of both PTEN and SAV1 (Figure 3, C and D, and Supplemental Figure 3, A and B), and decreased the mRNA levels of the known CEBPA-target genes Bcl-2 and p21 (refs. 22, 23, and Supplemental Figure 3C), suggesting that CEBPA positively regulates PTEN and SAV1 in PACs. In silico analysis indicated that there are several CEBPA binding sites in their promoter regions, and a ChIP assay showed that CEBPA proteins bind to these regions in 266-6 cells (Supplemental Figure 3D), indicating that Cebpa transcriptionally regulates the Pten and $S a v 1$ genes by binding to their promoters in pancreatic cells. CEBPA knockdown also substantially increased activating phosphorylation of AKT and decreased inhibitory phosphorylation of YAP in 266-6 cells (Figure 3E and Supplemental Figure 3E). Moreover, CEBPA knockdown markedly increased SOX9 mRNA and protein levels in conjunction with CTGF upregulation in 266-6 cells (Figure 3, C and E, and Supplemental Figure 3, A and E). To investigate whether the phenotypic changes induced by CEBPA knockdown in 266-6 cells are mediated by the decreased expression levels of both PTEN and SAV, we examined the effect of PTEN and SAV1 re-expression on the ADM phenotype of CEBPA-silenced cells in vitro. We confirmed that the transfection of Pten and Sav1 cDNA increased the protein levels of both PTEN and SAV1 in 266-6 cells (Supplemental Figure 3F) and significantly suppressed the increase in the mRNA levels of Ctgf and Sox 9 upon CEBPA knockdown (Supplemental Figure 3G), suggesting that the decrease in PTEN and SAV1 resulting from CEBPA inhibition induces ADM in vitro. Furthermore, CTGF inhibition significantly suppressed the increase in Sox9 levels in 266-6 PACs with CEBPA knockdown (Figure $3 \mathrm{~F}$ and Supplemental Figure $3 \mathrm{H}$ ). Taken together, these data suggested that CEBPA inhibition reduced PTEN and SAV1, which induced transdifferentiation of PACs through CTGF upregulation. We also searched for upstream signals that triggered CEBPA downregulation in CP models. We found that expression levels of the Hnf $4 a$ and Cebpb genes, both of which are reported to positively regulate Cebpa $(24,25)$, significantly decreased in both CP models compared with controls (Supplemental Figure 3, I and J).

Inhibition of PTEN and SAV1 in PACs may activate surrounding macrophages and PSCs via CTGF upregulation in vitro. To further examine the mechanisms underlying $\mathrm{CP}$ development in the absence of PTEN and SAV1, we focused on the interaction between PACs and macrophages/PSCs. First, we examined the expression levels of inflammatory cytokines in the macrophage cell line RAW 264.7 two days after coculture in a Transwell system with 266-6 PACs transfected with Cebpa siRNA or negative control siRNA. CEBPA knockdown in acinar cells significantly increased the mRNA expression levels of Tnfa, Illb, and Ccl2 in the cocultured RAW 264.7 cells (Figure 4A and Supplemental Figure 4A). Next, we cocultured 266-6 PACs and PSCs isolated from mouse pancreata in the Transwell coculture system. The mRNA expression levels of Tgfb1, Col1a1, and Col1a2 were significantly higher in PSCs cocultured with 266-6 cells with CEBPA knockdown than in PSCs cocultured with 266-6 cells transfected with negative control siRNA (Figure 4B and Supplemental Figure 4B). These results suggest that PACs with CEBPA knockdown-mediated inhibition 
A

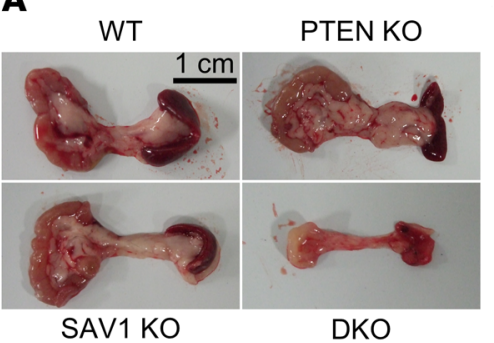

SAV1 KO

D

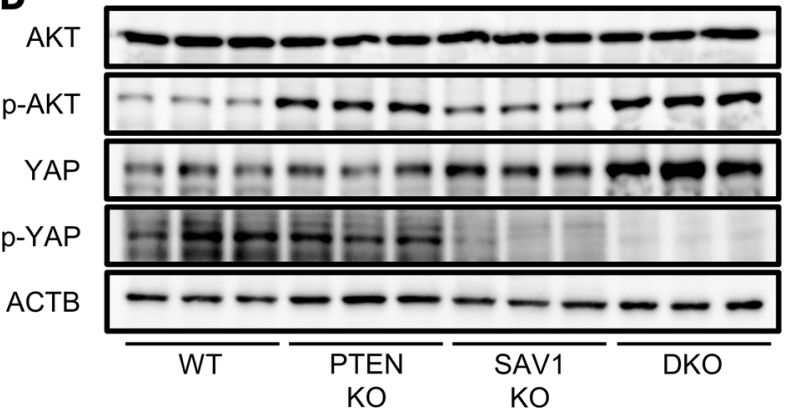

B

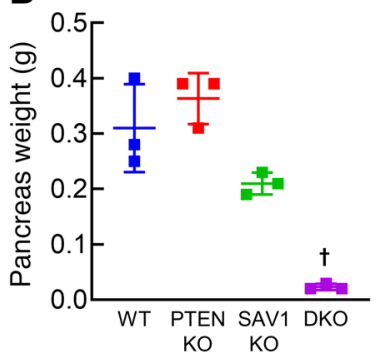

C

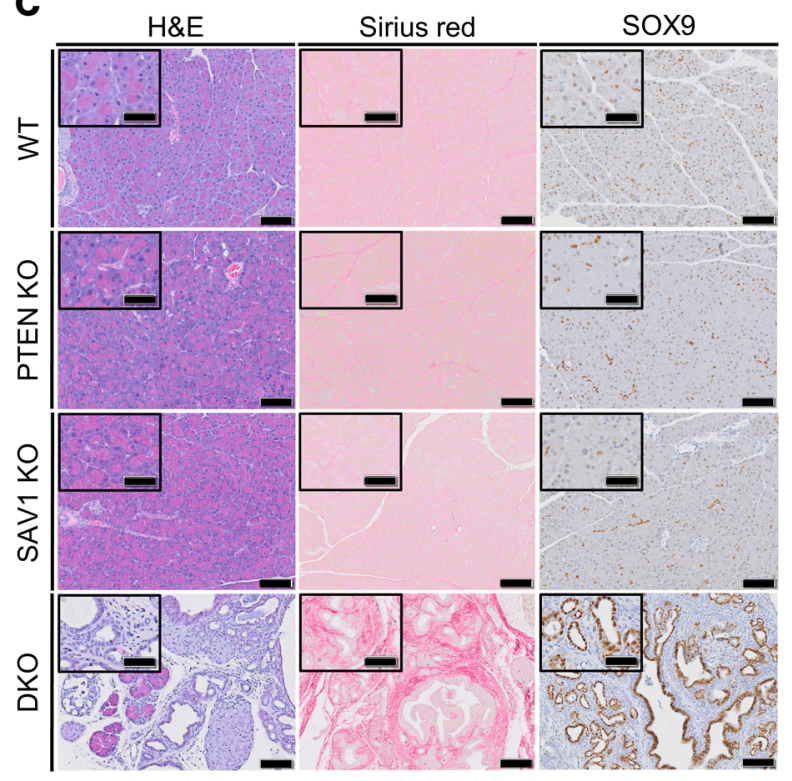

E Cd68

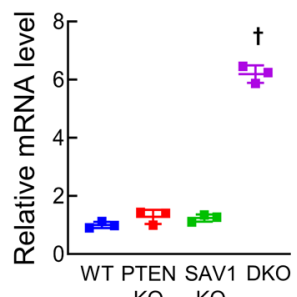

Tgfb1

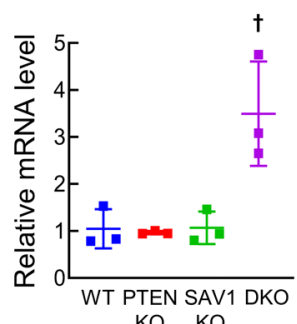

Tnfa

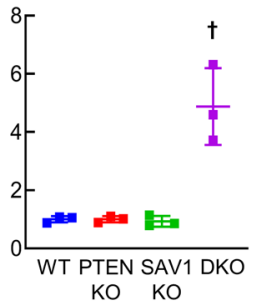

Col1a1

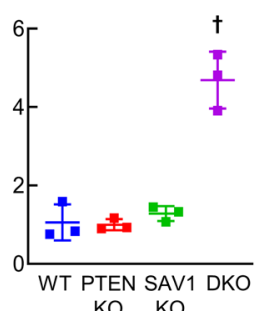

$1 / 1 b$

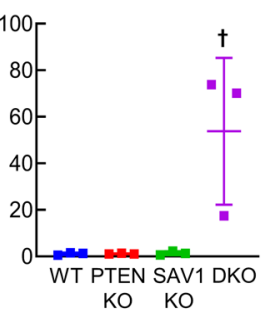

Col1a2

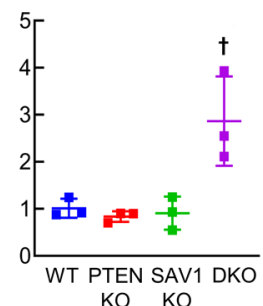

Ccl2

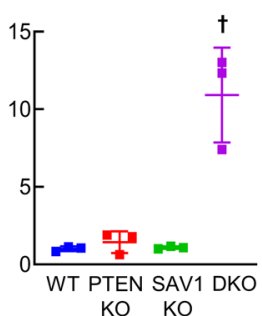

Ctgf

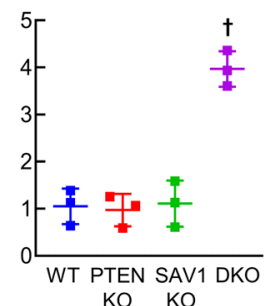

Figure 2. Mice with pancreas-specific loss of Pten and Sav1 spontaneously develop CP. Pancreatic phenotypes were examined in WT mice, pancreasspecific Pten knockout (PTEN-KO) mice, Sav1-knockout (SAV1-KO) mice, and Pten and Sav1 double knockout (DKO) mice at 6 weeks of age. (A) Macroscopic images of the pancreas. (B) Pancreas weight. (C) Representative images of H\&E, Sirius red, and SOX9 staining of pancreatic tissue. (D) Protein levels of AKT, p-AKT, YAP, p-YAP, and ACTB in the pancreata of mice. (E) Cd68, Tnfa, I11b, Ccl2, Tgfb1, Col1a1, Col1a2, and Ctgf mRNA levels in pancreatic tissue. Blots run in parallel contemporaneously are shown. All data are presented as the means \pm SDs of results for 3 mice per group. One-way ANOVA with Tukey's post hoc test was used to compare differences among 4 groups. ${ }^{\dagger} P<0.05$ vs. all groups. Scale bars: $100 \mu \mathrm{m}$ and $50 \mu \mathrm{m}$ (insets).

of PTEN and SAV1 may activate surrounding macrophages and PSCs. Interestingly, further CTGF inhibition in 266-6 PACs with CEBPA knockdown significantly suppressed the increase in the expression levels of these inflammatory cytokines in cocultured RAW 264.7 cells (Figure 4C and Supplemental Figure 4C) and prevented upregulation of profibrogenic genes in cocultured PSCs (Figure 4D and Supplemental Figure 4D), suggesting that inhibition of PTEN and SAV1 in PACs may activate surrounding macrophages and PSCs via CTGF upregulation in vitro.
CTGF produced by PACs is involved in the development of CP via pancreas-specific loss of Pten and Sav1 in vivo. To investigate whether CTGF derived from PACs is involved in CP induced by deletion of Pten and Sav1 in mice, we generated mice with pancreas-specific triple knockout of Pten, Sav1, and Ctgf (Pdx1-Cre Pten ${ }^{f / f l} \operatorname{Sav1}^{7 / f l}$ Ctgfl/f [triple KO; TKO] mice) and compared pancreatic phenotypes among WT, DKO, and TKO mice. We first confirmed that the levels of Pten, Sav1, and Ctgf were significantly decreased in the pancreata of TKO mice compared with WT mice (Figure 5A). The 
A

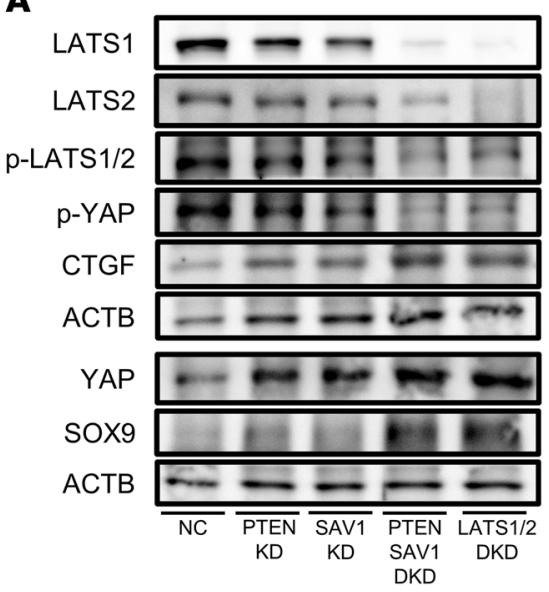

B

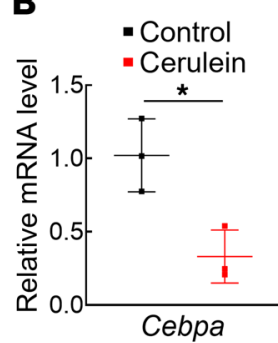

D

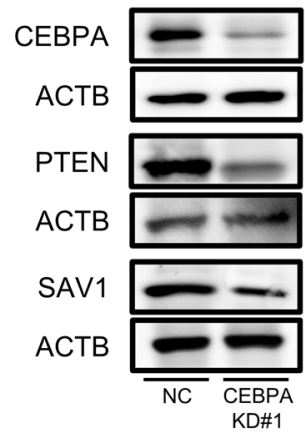

C
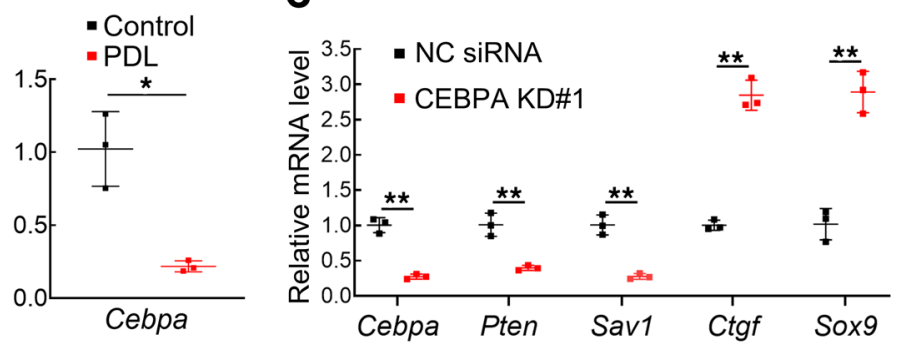

$\mathbf{E}$

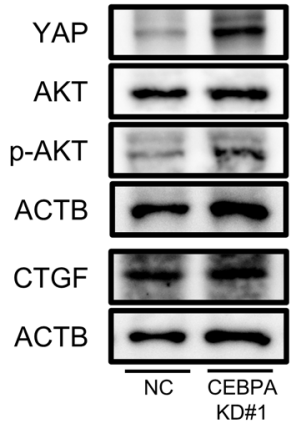

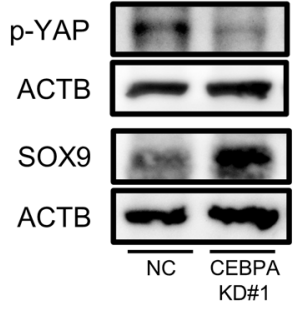
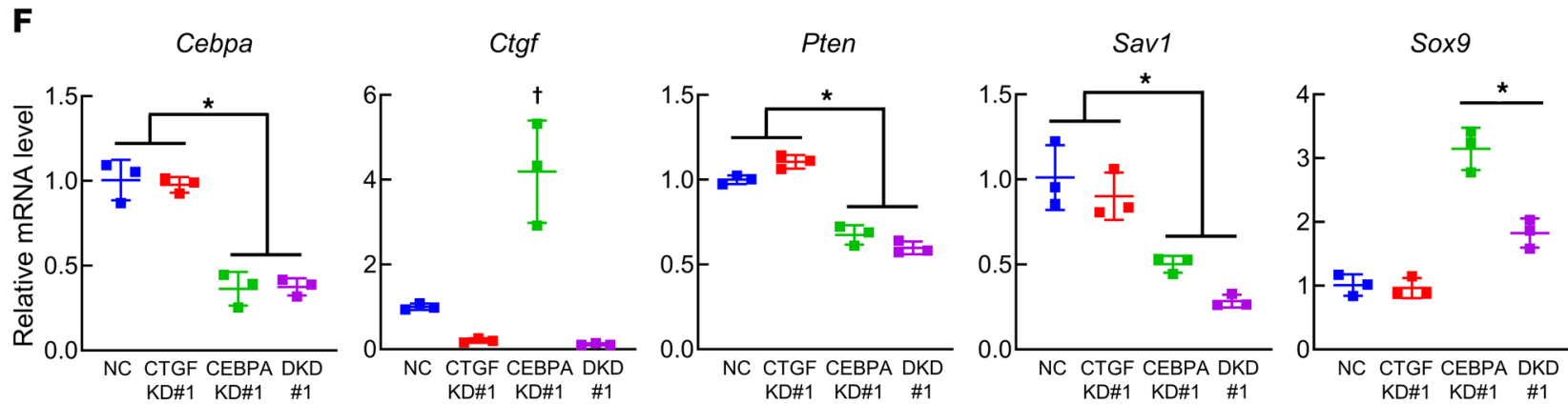

Figure 3. CEBPA positively regulates PTEN and SAV1 in acinar cells, and inhibition of PTEN and SAV1 induces ADM through CTGF upregulation in vitro. (A) Protein levels of LATS1, LATS2, p-LATS1/2, YAP, p-YAP, CTGF, SOX9, and ACTB in 266-6 cells 3 days after transfection with negative control (NC) siRNA, Pten siRNA (PTEN knockdown [KD]), Sav1 siRNA (SAV1 KD), both Pten and Sav1 siRNAs (PTEN SAV1 double KD [DKD]), or both Lats1 and Lats2 siRNAs (LATS1/2 DKD). (B) Cebpa mRNA levels in pancreatic tissue in mice after repeated cerulein or vehicle (control) injections (left) and in mice subjected to PDL surgery or sham surgery (right). (C and D) mRNA levels of Cebpa, Pten, Sav1, Ctgf, and Sox9 (C) and protein levels of CEBPA, PTEN, SAV1, and ACTB (D) in 266-6 cells 3 days after transfection with NC siRNA or Cebpa siRNA (\#1) (CEBPA KD\#1). (E) Protein levels of YAP, p-YAP, AKT, p-AKT, CTCF, SOX9, and ACTB in 266-6 cells 3 days after transfection with NC siRNA or Cebpa siRNA (\#1) (CEBPA KD\#1). (F) mRNA levels of Cebpa, Ctgf, Pten, Sav1, and Sox9 in 266-6 cells 3 days after transfection with NC siRNA, Ctgf siRNA (\#1) (CTGF KD\#1), Cebpa siRNA (\#1) (CEBPA KD\#1), or both Cebpa (\#1) and Ctgf siRNAs (\#1) (DKD\#1). Blots run in parallel contemporaneously or run at different times with loading control for each gel are shown. The data are presented as the means \pm SDs of results for 3 samples per group. Student's $t$ test was used to evaluate differences between 2 groups (B and $\mathbf{C})$. One-way ANOVA with Tukey's post hoc test was used to compare differences among 4 groups $(\mathbf{F})$. ${ }^{*} P<$ $0.05,{ }^{* *} P<0.005$, and ${ }^{\dagger} P<0.05$ vs. all groups.

weight of the pancreas was significantly increased in TKO mice compared with DKO mice (Figure 5, B and C). Importantly, histological analysis showed that the acinar cell loss, ADM formation, and fibrosis observed in DKO mice were mitigated in TKO mice (Figure 5, D and E). In addition, the pancreatic expression levels of $C d 68$, together with those of Tnfa, Illb, and Ccl2, were significantly lower in TKO mice than in DKO mice (Figure 5F). Similarly, Ctgf deletion significantly suppressed the upregulation of Tgfb1, Col1a1, and Col1a2 expression observed in DKO mice (Figure 5F). While all DKO mice died early (Supplemental Figure 2F), some of the TKO mice survived to 10 months of age without developing PanIN or PDAC (Supplemental Figure 5A). Taken together, these results indicate that in the absence of PTEN and SAV1, CTGF in PACs promotes $\mathrm{CP}$ development in mice via the acceleration of $\mathrm{ADM}$ formation and activation of surrounding macrophages and PSCs.

CTGF inhibition ameliorates CP by alleviating inflammation, fibrogenesis, and ADM formation in vivo. To explore the therapeutic potential of targeting CTGF in CP, we treated mice with cerulein-induced CP with either FG-3154, an anti-CTGF neutralizing antibody, or control IgG. The therapeutic protocol is shown in Figure 6A. Compared with control IgG treatment, FG-3154 treatment significantly alleviated the pancreatic weight loss caused by repeated cerulein injection (Figure 6, B and C). Histologically, while $\mathrm{CP}$ post-treatment phenotypes were comparable to pre- 
A

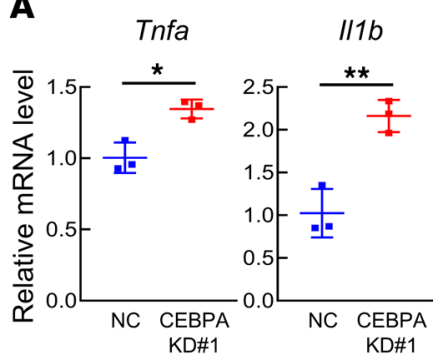

Ccl2 RAW 264.7 cells cocultured with - 266-6 + NC siRNA

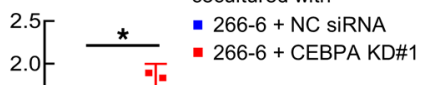

\section{B}
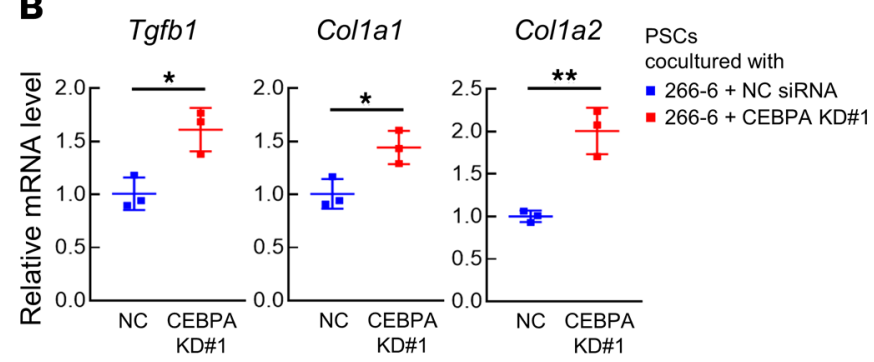
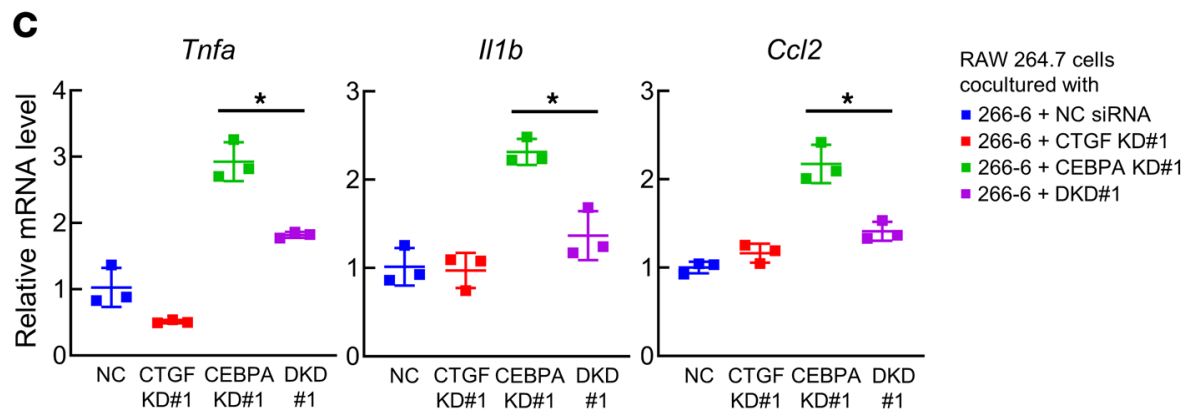

D

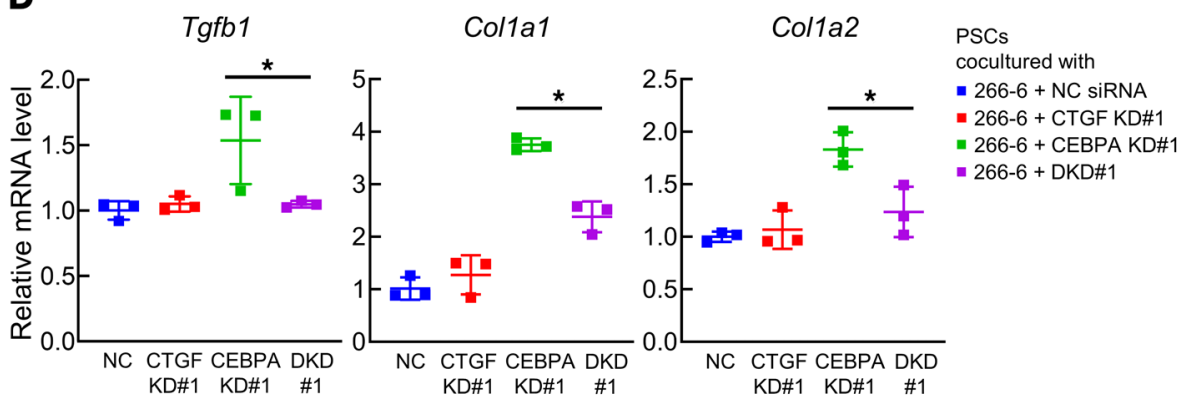

Figure 4. Inhibition of PTEN and SAV1 in PACs may activate surrounding macrophages and PSCs via CTGF upregulation in vitro. (A) mRNA levels of Tnfa, II1b, and Ccl2 in RAW 264.7 cells 2 days after coculture with 266-6 cells transfected with negative control (NC) siRNA or Cebpa siRNA (\#1) (CEBPA knockdown [KD] \#1). (B) mRNA levels of Tgfb1, Col1a1, and Col1a2 in PSCs isolated from mouse pancreata 2 days after coculture with 266-6 cells transfected with NC siRNA or Cebpa siRNA (\#1) (CEBPA KD\#1). (C) mRNA levels of Tnfa, II1b, and Ccl2 in RAW 264.7 cells 2 days after coculture with $266-6$ cells transfected with NC siRNA, Ctgf siRNA (\#1) (CTCF KD\#1), Cebpa siRNA (\#1) (CEBPA KD\#1), or both Cebpa (\#1) and Ctgf (\#1) siRNAs (double KD [DKD] \#1). (D) mRNA levels of Tgfb1, Col1a1, and Col1a2 in PSCs isolated from mouse pancreata 2 days after coculture with 266-6 cells transfected with NC siRNA, Ctgf siRNA (\#1) (CTGF KD\#1), Cebpa siRNA (\#1) (CEBPA KD\#1), or both Cebpa (\#1) and Ctgf (\#1) siRNAs (DKD\#1). All data are presented as the means \pm SDs of results for 3 samples per group. Student's $t$ test was used to evaluate differences between 2 groups (A and B). One-way ANOVA with Tukey's post hoc test was used to compare differences among 4 groups (C and $\mathbf{D}) .{ }^{*} P<0.05$ and ${ }^{* *} P<0.005$.

treatment phenotypes in the control IgG group (Figure 6, D and E), the anti-CTGF neutralizing antibody treatment alleviated CP phenotypes (Figure 6, D and E). Furthermore, compared with control IgG treatment, FG-3154 treatment significantly suppressed the upregulation of Cd68, Tnfa, Illb, Ccl2, Tgfb1, Col1a1, and Col1a 2 expression caused by repeated cerulein injection (Figure 6F). Taken together, our data show that CTGF inhibition suppresses $\mathrm{CP}$ progression by alleviating inflammation, fibrogenesis, and ADM formation, thereby suggesting the targeting of CTGF as a novel potential therapeutic approach for $\mathrm{CP}$.

CP tissues exhibit lower CEBPA, PTEN, and SAV1 levels and higher CTGF levels than nonpancreatitis tissues from humans. Finally, we assessed the clinical significance of our findings using CP tissues and nonpancreatitis tissues from patients. To this end, we first histopathologically evaluated obstructive pancreatitis tissues and nonpancreatitis tissues from the same patients. Among patients who underwent surgical resection of the pancreas due to PDAC development at the pancreatic body, 6 exhibited obstructive pancreatitis on the tail side but not the head side. CP tissues exhibited decreased numbers of PACs, increased areas of fibrosis, and SOX9-positive ADM lesions compared with nonpancreatitis tissues in these patients (Figure 7, A and B). We then performed immunohistochemical staining for CEBPA, PTEN, SAV1, phosphorylated AKT (p-AKT), YAP, and CTGF in the pancreatic tissues. The expression levels of PTEN, SAV1, and CEBPA were significantly lower in CP tissues than in nonpancreatitis tissues (Figure 7, C and D). In contrast, p-AKT, nuclear YAP, and CTGF levels were significantly higher in CP tissues than in nonpancreatitis tissues (Figure 7, C and D). Next, we also performed the same histopathological analyses using pancreatic tissues from $\mathrm{CP}$ patients and normal pancreatic tissues from pancreatic neuroendocrine tumor patients. 
A

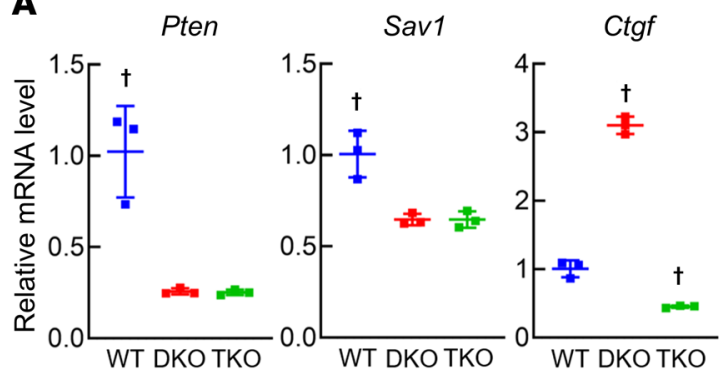

B

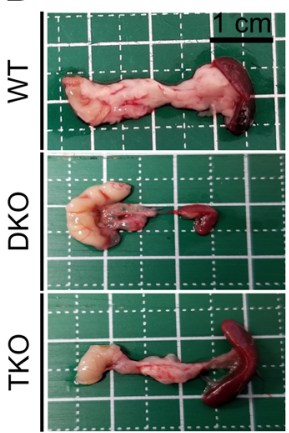

E

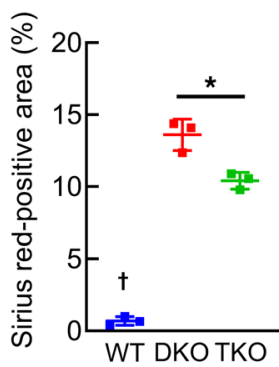

C

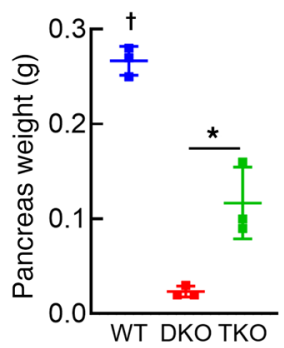

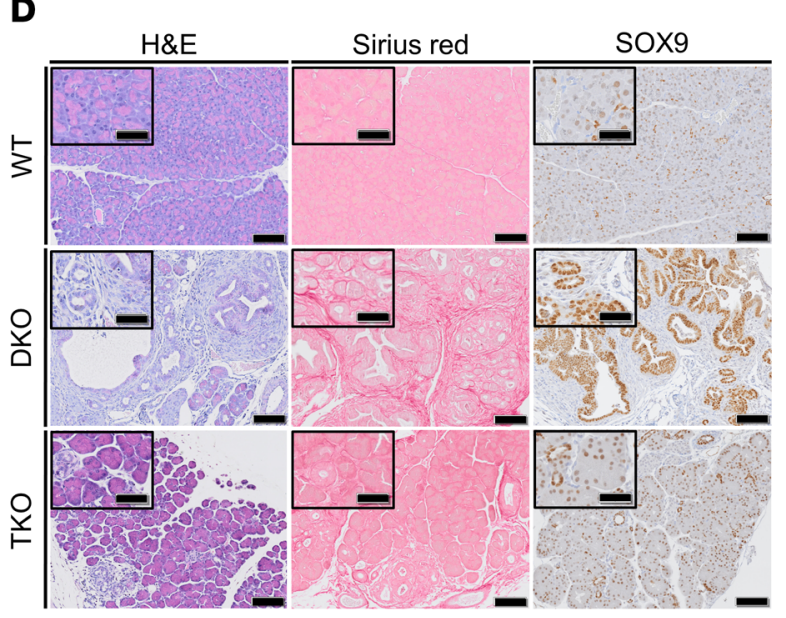

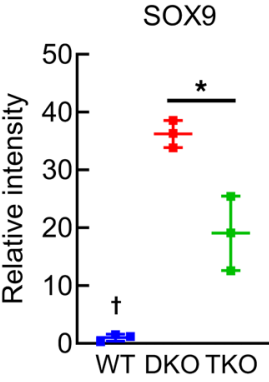

$\mathbf{F}$

Cd68

Tnfa

$/ / 1 b$
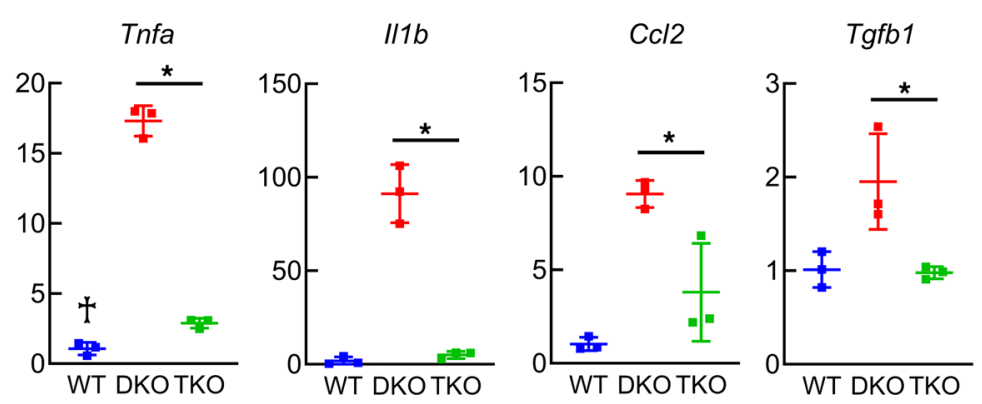

WT DKOTKO

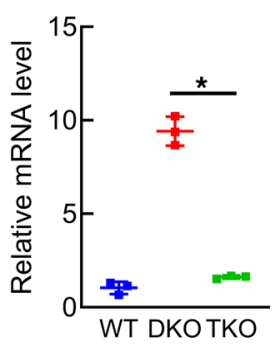

Figure 5. CTGF produced by PACs is involved in the development of CP via pancreas-specific Pten and Sav1 loss in vivo. Pancreatic phenotypes were examined in WT mice, mice with pancreas-specific Pten and Sav1 double knockout (DKO), and mice with Pten, Sav1, and Ctgf triple knockout (TKO) at 6 weeks of age. (A) Pten, Sav1, and Ctgf mRNA levels in pancreatic tissue. (B) Macroscopic images of the pancreas. (C) Pancreas weight. (D and E) Representative images of H\&E, Sirius red, and SOX9 staining of pancreatic tissue (D) and quantification of the Sirius red-positive area (E, left) and SOX9 staining intensity (E, right). (F) Cd68, Tnfa, I/1b, Ccl2, Tgfb1, Col1a1, and Col1a2 mRNA levels in pancreatic tissue. All data are presented as the means \pm SDs of results for 3 mice per group. One-way ANOVA with Tukey's post hoc test was used to compare differences among 3 groups. ${ }^{*} P<0.05$ and ${ }^{\dagger} P<0.05$ vs. all groups. Scale bars: $100 \mu \mathrm{m}$ and $50 \mu \mathrm{m}$ (insets).

Similarly to the obstructive pancreatitis tissues, CP tissues showed significantly lower levels of PTEN, SAV1, and CEBPA and higher levels of p-AKT, nuclear YAP, and CTGF than those in normal pancreatic tissues (Figure 7E and Supplemental Figure $\left.6, \mathrm{~A}^{-} \mathrm{C}\right)$. Collectively, these data suggest that dysregulation of the CEBPA-PTEN/SAV1-CTGF axis might be involved in the development of $\mathrm{CP}$ in humans.

\section{Discussion}

In this study, we focused on the PI3K and Hippo signaling pathways, both of which are important for PDAC development, and provided important in vivo experimental evidence that dysregulation of these pathways synergistically contributes to $\mathrm{CP}$ pathogenesis. Additionally, we found that downregulation of PTEN and SAV1 is partially responsible for dysregulation of these pathways and occurs in human CP patients, supporting the clinical relevance of our findings. Recently, crosstalk between the PI3K and Hippo signaling pathways has been shown to be involved in organ development as well as in the pathophysiology of various diseases (2629). During organ development, YAP downregulates PTEN via microRNA-29 induction and activates PI3K-mTOR signaling, regulating organ size (30). Inversely, PTEN inactivation suppresses 
A

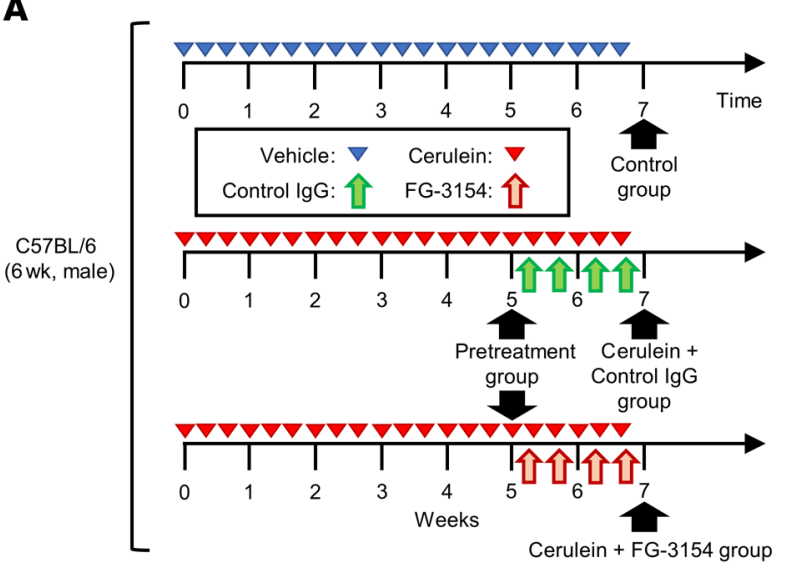

B

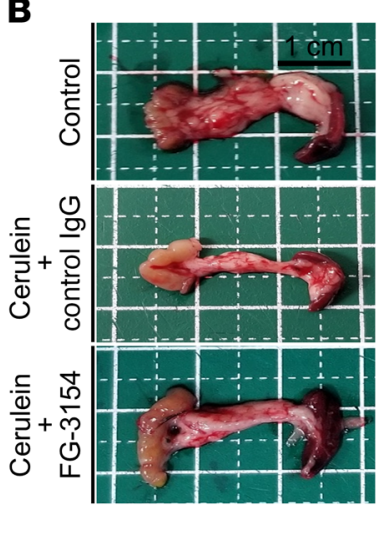

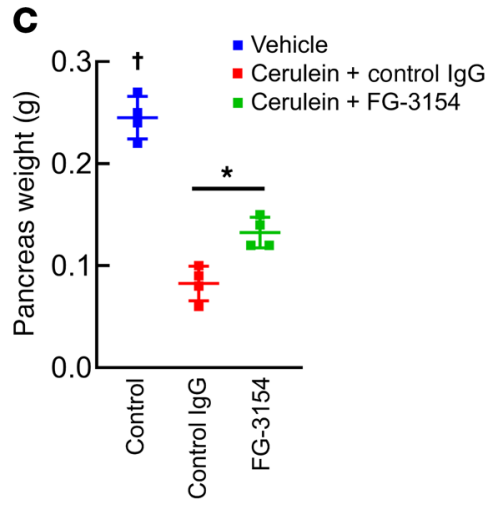

D

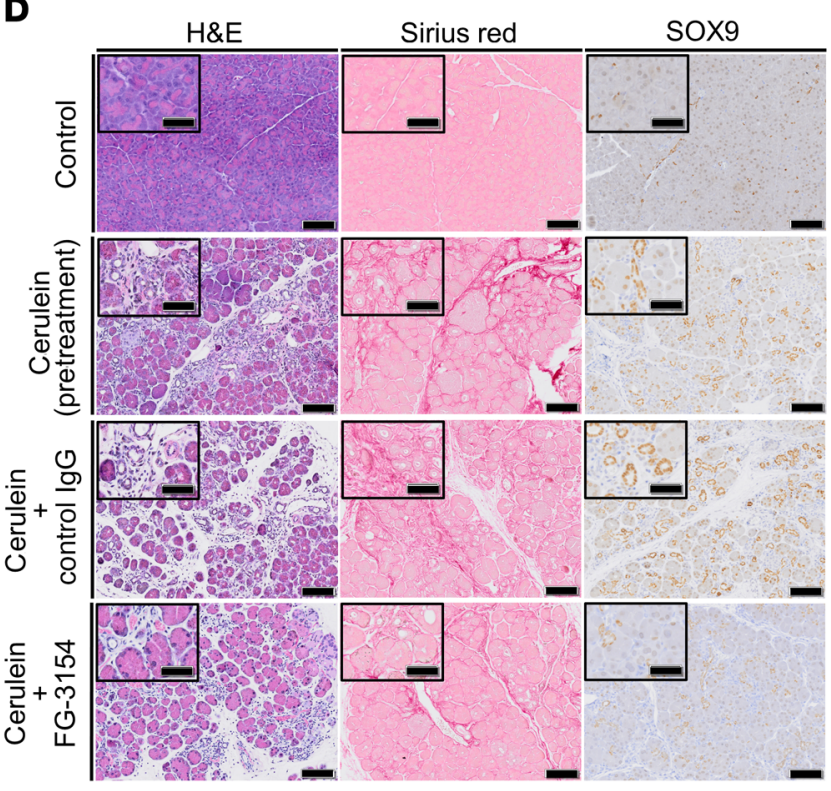

E

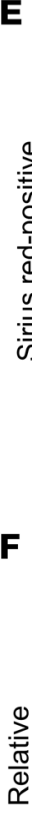

\section{F}

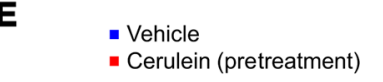

- Cerulein (pretreatment)
- Cerulein + control lgG - Cerulein + FG-3154
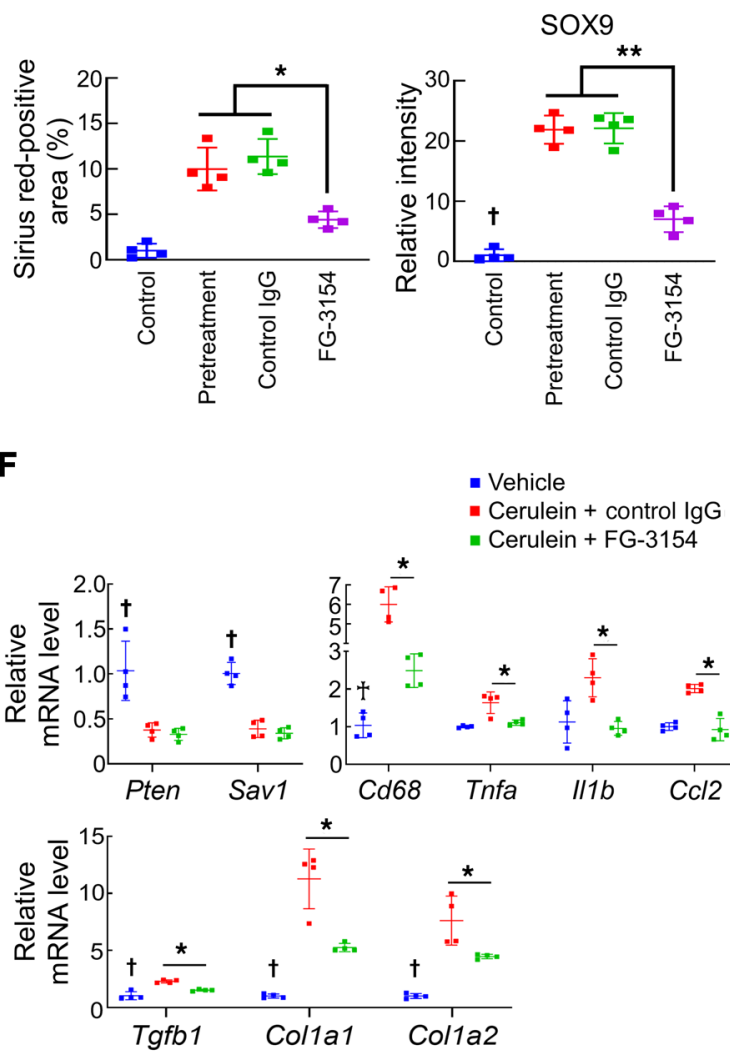

Figure 6. CTGF inhibition ameliorates CP by alleviating inflammation, fibrogenesis, and ADM formation in vivo. Pancreatic phenotypes were examined in vehicle-treated mice (control) and mice with cerulein-induced CP upon treatment with control lgG or FG-3154 (40 mg/kg) twice weekly for 2 weeks. (A) Therapeutic protocol. (B) Macroscopic images of the pancreas. (C) Pancreas weight. (D and E) Representative images of H\&E, Sirius red, and SOX9 staining of pancreatic tissue (D) and quantification of the Sirius red-positive area (E, left) and SOX9 staining intensity (E, right). (F) Pten, Sav1, Cd68, Tnfa, I/1b, Ccl2, Tgfb1, Col1a1, and Col1a2 mRNA levels in pancreatic tissue. All data are presented as the means \pm SDs of results for 4 mice per group. One-way ANOVA with Tukey's post hoc test was used to compare differences among 3 or 4 groups. ${ }^{*} P<0.05$, ${ }^{* *} P<0.005$, and ${ }^{\dagger} P<0.05$ vs. all groups. Scale bars: $100 \mu \mathrm{m}$ and $50 \mu \mathrm{m}$ (insets).

the MOB1-LATS1/2 interaction, leading to YAP activation, which accelerates gastric tumorigenesis (28). Therefore, these pathways are regulated bidirectionally at the molecular level. Moreover, we previously showed that simultaneous loss of PTEN and SAV1 in the liver induces synergistic activation of liver progenitor cells, leading to early and aggressive hepatocarcinogenesis (29), indicating the presence of synergistic crosstalk between these pathways. In the present study, we observed that single deletion of either Pten or Sav1 did not result in any pancreatic phenotype, but dual deletion resulted in rapid disruption of exocrine homeostasis, leading to $\mathrm{CP}$ development. Therefore, synergistic crosstalk between the PI3K and Hippo signaling pathways also exists in the pancreas and 

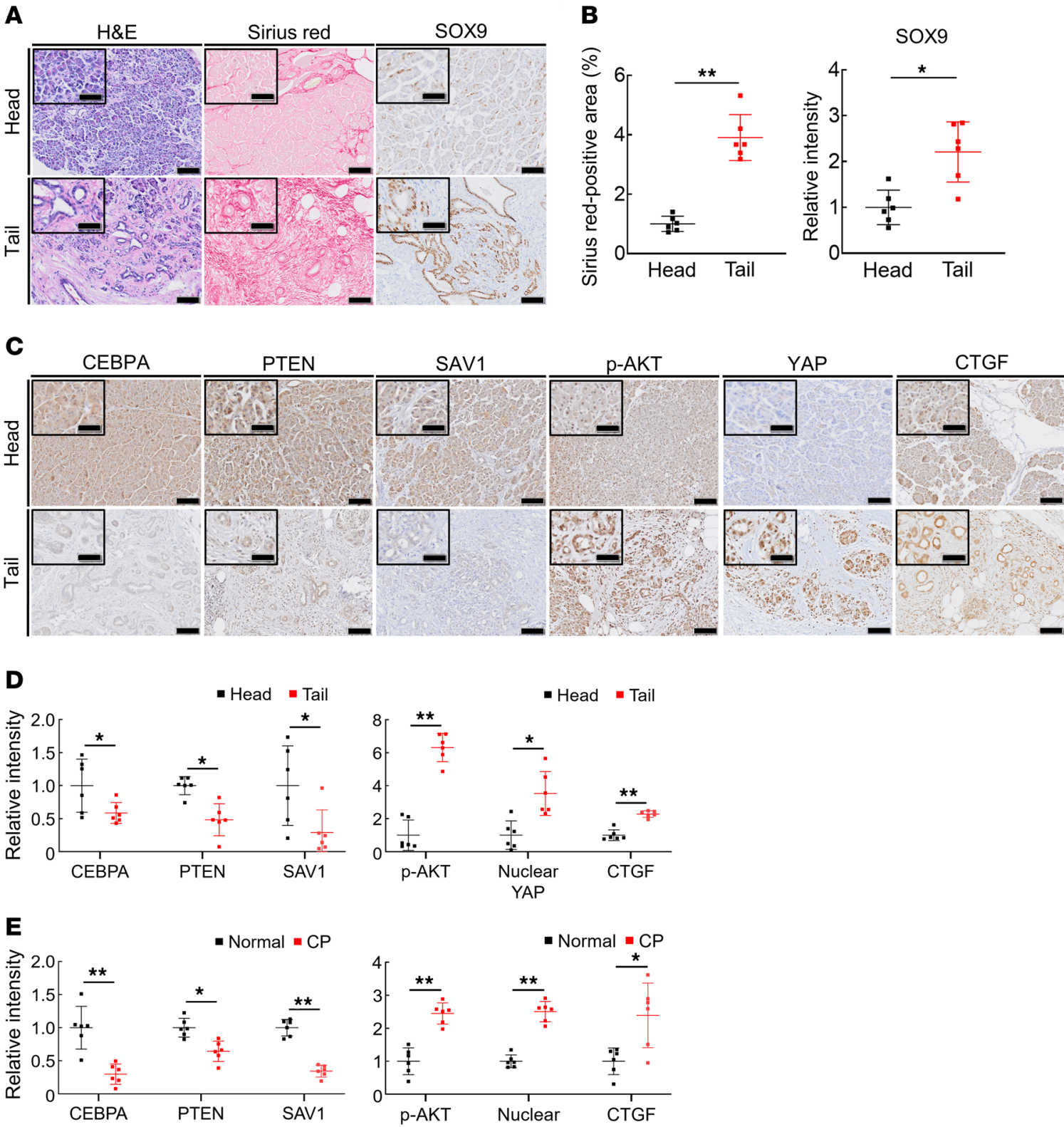

Figure 7. CP tissues exhibit lower PTEN and SAV1 levels and higher CTGF levels than nonpancreatitis tissues from humans. (A and B) Representative images of H\&E, Sirius red, and SOX9 staining of pancreatic tissue from the head or tail side of PDAC tumors from pancreatic cancer patients (A) and quantification of the Sirius red-positive area (B, left) and SOX9 staining intensity (B, right). (C and $\mathbf{D})$ Representative images of CEBPA, PTEN, SAV1, p-AKT, nuclear YAP, and CTCF staining of pancreatic tissue from the head or tail side of PDAC tumors from pancreatic cancer patients (C) and the relative staining intensity of CEBPA, PTEN, SAV1, p-AKT, nuclear YAP, and CTCF (D). (E) The relative intensity of CEBPA, PTEN, SAV1, p-AKT, nuclear YAP, and CTCF staining of pancreatic tissue from the normal pancreatic regions obtained from pancreatic neuroendocrine tumor patients or from the chronic pancreatic regions obtained from CP patients. All data are presented as the means \pm SDs of results for 6 samples per group. Student's $t$ test was used to evaluate differences between 2 groups. ${ }^{*} P<0.05$ and ${ }^{*} P<0.005$. Scale bars: $100 \mu \mathrm{m}$ and $50 \mu \mathrm{m}$ (insets).

plays a critical role in $\mathrm{CP}$ pathogenesis. At the cellular and molecular levels, we found that either SAV1 or PTEN inhibition slightly reduced phosphorylation of LATS1/2, leading to only mild YAP activation in PACs. In contrast, dual deletion of SAV1 and PTEN almost completely eliminated the phosphorylation of LATS1/2 and thus strongly activated YAP, inducing ADM in these cells. These data suggested that crosstalk between PTEN and SAV1 affected the activation of LATS1/2-YAP signaling in PACs. This YAP activity was comparable to that acquired through LATS1/2 inhibition (Figure 3A). This may reflect the previous report that mice with deletion of both Lats1 and Lats 2 spontaneously develop $\mathrm{CP}$ similar to that in our DKO mice (31).

In an aim to identify the upstream regulator of the PI3K and Hippo signaling pathways in acinar cells, we found that the expression level of CEBPA decreased along with the expression levels of both PTEN and SAV1 in the pancreata of mice with $\mathrm{CP}$ induced via either of 2 different methods. CEBPA, a member of the C/EBP family of TFs, arrests cellular proliferation 
and drives terminal differentiation in a variety of adult tissues, including those composed of granulocytes, adipocytes, hepatocytes, pneumocytes, and osteoclasts $(23,32,33)$. In addition, CEBPA is indispensable for maintaining postnatal systemic energy homeostasis and lipid storage (34). The CEBPA gene has been reported to act as a tumor suppressor in many tumor types and to be epigenetically silenced in pancreatic cancer cells (23, 35). On the other hand, the functional role of CEBPA in PACs remains unclear. CEBPA directly controls many genes involved in the cell cycle and cell proliferation, such as CDKN1A, E2F1, and $M Y C$, as well as a variety of metabolic genes $(23,34)$. In this study, we demonstrated, for the first time to our knowledge, that CEBPA positively regulates PTEN and SAV1 in PACs and maintains their integrity by preventing their transformation into ductal cells. Considering that CEBPA positively regulates 2 major tumor suppressors in the pancreas, further examination of the involvement of this tumor-suppressive link in PDAC development would be interesting.

We focused on CTGF as the critical downstream mediator of the PI3K and Hippo pathways during CP development. Several studies have shown that CTGF acts as a profibrogenic factor and activates fibroblasts in various organs including the pancreas, facilitating fibroblast proliferation and collagen production, which promote organ fibrogenesis $(15,36-38)$. CTGF is considered to be produced mainly by fibroblasts, but recent reports have shown that CTGF secreted from epithelial cells also contributes significantly to disease pathogenesis $(39,40)$. We and others have reported that CTGF secreted from hepatocytes may contribute to liver fibrogenesis via activation of nearby hepatic stellate cells $(39,41,42)$. In the pancreas, Charrier et al. reported that CTGF was produced from PACs, as well as PSCs, under ethanol stimulus, suggesting the potential involvement of PAC-derived CTGF in CP development (43). In our current study, we found that in vitro, PAC-derived CTGF promotes ductal metaplasia and activates surrounding macrophages and PSCs, suggesting a novel pleiotropic function of PAC-derived CTGF during CP development in cell-autonomous and cell-heteronomous manners. Furthermore, we demonstrated, for the first time to our knowledge, that PAC-derived CTGF is involved in $\mathrm{ADM}$ formation, inflammation, and fibrogenesis during $\mathrm{CP}$ in vivo. These results suggest that PAC-derived CTGF is pivotal in the development of CP.

CTGF expression has previously been reported to be increased in human CP (44). However, its regulatory mechanism and pathological significance remain unclear. In this study, we showed that genetic disruption of Pten and Sav1 synergistically upregulates CTGF expression in the pancreata of mice with $\mathrm{CP}$, providing, to our knowledge, the first demonstration of the regulatory link between PTEN/SAV1 and CTGF in CP. Consistent with this finding, we also observed that in human obstructive pancreatitis, the expression of PTEN and SAV1 was decreased, whereas that of CTGF was increased (44), suggesting the existence of this regulatory link in human CP. More importantly, we demonstrated the in vivo therapeutic efficacy of blocking CTGF in mouse models of both genetically and chemically induced CP. Liu et al. also very recently showed the antifibrotic effects of an anti-CTGF neutralizing antibody in a different animal model of CP, Lats1/2-double-knockout mice (31). Taken together, the results of our studies highlight CTGF as a promising therapeutic target for CP. Moreover, we showed that in the cerulein-induced murine model of CP, treatment with an anti-CTGF neutralizing antibody alleviated inflammation, fibrosis, and ADM, all of which are known to induce PDAC development. Therefore, amelioration of inflammation and fibrosis during CP via CTGF inhibition might also prevent CP-induced pancreatic carcinogenesis, and this important beneficial effect needs further assessment.

In conclusion, our study revealed the important molecular mechanism of CP development, i.e., that dysregulation of PI3K and Hippo signaling in PACs promotes ADM, which is followed by inflammation with macrophage infiltration and fibrosis with PSC activation, resulting in rapid development of CP. Furthermore, we identified CTGF as the downstream effector of PI3K and Hippo signaling responsible for CP progression and provided robust evidence that $\mathrm{CTGF}$ inhibition reduces the severity of $\mathrm{CP}$ in vivo. These results indicate that targeting CTGF could be an effective therapeutic strategy for CP.

\section{Methods}

Mice. C57BL/6J and Pten ${ }^{f /+}$ mice were purchased from The Jackson Laboratory. Pdx1-Cre transgenic mice were obtained from the Mouse Models of Human Cancer Consortium (National Cancer Institute-Frederick, Bethesda, Maryland). $S a v 1^{f l / f l}$ and $C t g f^{l / f l}$ mice were generated as previously described $(45,46)$. To establish the model of cerulein-induced CP, 6-week-old C57BL/6J mice received cerulein (solubilized in PBS at a final concentration of $10 \mu \mathrm{g} / \mathrm{mL}$ and injected i.p. at a dose of $50 \mu \mathrm{g} / \mathrm{kg}$ ) every hour for 6 hours 3 times per week for 7 weeks. Controls received PBS injections. To establish the model of pancreatic duct ligation-induced CP, 6-week-old C57BL/6J mice were subjected to pancreatic duct ligation as previously described (18). Controls were subjected to surgery without duct ligation. The mice were sacrificed at the indicated time points. To generate Pdx1-Cre Pten ${ }^{f / f l}$ Sav1 $^{f l / f l}$ mice, Pten ${ }^{f /+}$ mice or Sav1 $1^{f / f l}$ mice were first crossed with $P d x 1$-Cre mice to generate $P d x 1$-Cre $\mathrm{Pten}^{f /+}$ mice or Pdx1-Cre Sav1 $1^{f /+}$ mice. The resulting mice were then intercrossed to generate $\mathrm{Pten}^{f / f l} \mathrm{Sav1}^{f / f l}$ (WT) mice, Pdx1-Cre Pten $^{f / f l}$ (PTEN-KO) mice, Pdx1-Cre Sav1 ${ }^{f / f l}$ (SAV1-KO) mice, and Pdx1-Cre Pten ${ }^{f l / f l} S a v 1^{f l / f l}$ (DKO) mice. Additionally, the resulting mice were crossed with $C t g f^{l / f l}$ mice to generate Pdx1-Cre Pten ${ }^{f / f l}$ $S a v 1^{f l / f l} C_{t g} f^{f l / f l}$ (TKO) mice. All the genetically engineered mouse models were followed into adulthood.

Blood and tissue preparation. All mice were sacrificed under pentobarbital anesthesia, and peripheral blood and samples of the pancreas were collected for study. Harvested blood was centrifuged at $16,500 \mathrm{~g}$ for 10 minutes to separate the serum. Each pancreas was rapidly removed, weighed, and either snap-frozen for molecular analysis or fixed with $10 \%$ neutral phosphate-buffered formalin for histological analysis.

Histological and immunohistochemical analyses. Pancreatic tissues were stained with a standard H\&E preparation. Sirius red staining was performed to assess the degree of pancreatic fibrosis. To detect apoptotic cells, a TUNEL assay was performed with an ApopTag Peroxidase In Situ Apoptosis Detection Kit according to the manufacturer's instructions (Merck $\mathrm{KGaA}$ ). Immunohistochemical analyses 
of paraffin-embedded pancreatic tissues were performed using antibodies specific for PTEN, SOX9, p-AKT, glucagon, CEBPA, and CTGF (Abcam); YAP and Ki67 (Cell Signaling Technology); and SAV1 (Novus Biologicals). Immunostained tissues were imaged with a SLIDEVIEW VS200 (Olympus), and the immunostaining intensity of each PAC was quantified with ImageJ software (NIH). Detailed antibody information is given in Supplemental Table 1.

Biochemical analysis. Mouse serum glucose levels were enzymatically measured at the Oriental Kobo Life Science Laboratory (Tokyo, Japan). Mouse serum insulin levels were measured using an ELISA kit (Morinaga Institute of Biological Science).

RNA isolation and quantitative PCR. The total RNA was extracted from cell lines or pancreatic tissues using an RNeasy Mini Kit (QIAGEN) according to the manufacturer's instructions, and cDNA was synthesized using reverse transcription as previously described (47). Quantitative PCR was performed in a Quant Studio 6 Flex RealTime PCR System (Thermo Fisher Scientific) with TaqMan probes. Relative gene expression levels were determined by the $\Delta \Delta \mathrm{CT}$ method and normalized to those of Actb. Detailed probe (Thermo Fisher Scientific) information is given in Supplemental Table 2.

Western blot analysis. Samples of pancreatic tissues or harvested cells were lysed in lysis buffer (1\% Nonidet P-40, 0.5\% sodium deoxycholate, $0.1 \%$ SDS, protease inhibitor cocktail [Nacalai Tesque], phosphatase inhibitor cocktail [Nacalai Tesque], and PBS [pH 7.4]). Equal amounts of protein were electrophoretically separated and subjected to Western blotting. The following antibodies were used as primary antibodies: anti-AKT, anti-p-AKT, anti-YAP, anti-p-YAP, anti-CEBPA, anti-SOX9, anti-LATS1, anti-PTEN, antiSAV1 (all from Cell signaling Technology), anti-CTGF (Abcam), anti-LATS2 and anti-p-LATS1/2 (Invitrogen), and anti-ACTB (Sigma-Aldrich). Detailed antibody information is given in Supplemental Table 1.

Cell culture. The mouse PAC tumor cell line 266-6 and the mouse macrophage cell line RAW 264.7 were obtained from the American Type Culture Collection cell bank and cultured in DMEM (Nacalai Tesque) containing 10\% heat-inactivated FCS, $100 \mathrm{U} / \mathrm{mL}$ penicillin, and $100 \mu \mathrm{g} / \mathrm{mL}$ streptomycin. Mouse PSCs were isolated from C57BL/6 mice by a previously described method with modifications (48). All cells were maintained at $37^{\circ} \mathrm{C}$ in a humidified atmosphere containing $5 \% \mathrm{CO}_{2}$. All cells were confirmed to be pathogen and mycoplasma free.

ChIP assay. First, 266-6 cells were prepared using the SimpleChIP Plus Enzymatic Chromatin IP kit (9005, Cell Signaling Technology). Nuclear extracts from 266-6 cells were cross-linked by the addition of formaldehyde, sonicated, and used for immunoprecipitation with the CEBPA rabbit polyclonal antibody (18311-1-AP, Proteintech). Reversecross-linked ChIP DNA samples were subjected to real-time PCR using the oligonucleotides specific to promoter regions of the murine Pten gene, 5'-AGGAACAGCTTGGGGACTCT-3' (sense) and 5'-CCGGATCGGAGCAGAGAATAG-3' (antisense), and to the murine Sav1 gene, $5^{\prime}$-TCTGGTGCTCAAAGGCTTCAA-3' (sense) and 5' -TCCGCGCTTTCGGATTAAA-3' (antisense), which were synthesized by Integrated DNA Technologies.

Cell transfection with siRNA. Silencer Select siRNAs against mouse Pten, Sav1, Lats1, Lats2, Cebpa, and Ctgf were designed and synthesized by Thermo Fisher Scientific. We used 2 independent siRNAs against Cebpa (\#1 and \#2) and against $\operatorname{Ctg} f(\# 1$ and \#2). Cells were transfected using the reverse transfection method. First, 2666 cells were transfected with siRNAs (10 nM) using Lipofectamine RNAiMAX (Thermo Fisher Scientific) according to the manufacturer's instructions. In brief, the 266-6 cells were plated in 12-well plates, and either Silencer Select negative control siRNAs or target siRNAs mixed with Lipofectamine RNAiMAX were added to the plates on the same day. Three to five days after transfection, the cells were used for experiments. Detailed siRNA information is given in Supplemental Table 3.

Plasmid construction and transfection. Pten and Sav1 expression vectors and 266-6 cells were used to establish transfectants of both of these genes. The full-length mouse Pten cDNA was synthesized by Integrated DNA Technologies. The LentiCas9-Blast plasmid (52962, Addgene) was cut with BamHI and XbaI restriction enzymes, and then the Pten cDNA was inserted into the plasmid. Additionally, the Sav1 expression vector was purchased from OriGene (MR206033L4). Accordingly, the 266-6 cells were then transfected with both the Pten cDNA insert vector and Sav1 expression vector or control vector and Lipofectamine 2000 (11668019, Thermo Fisher Scientific). Puromycin and blasticidin were used for the selection of both Pten- and Sav1-expressing transfectants.

Transwell assay. RAW 264.7 cells or PSCs were cultured in the bottom compartment of a 12-well Transwell cell culture system (pore size $3.0 \mu \mathrm{m}$; Corning), and 266-6 cells transfected with either Silencer Select negative control siRNAs or target siRNAs were cultured on the membranes of the Transwell cell culture inserts. After 2 days of coculture in the Transwell system, the RAW 264.7 cells or PSCs were used for experiments.

Administration of FG-3154. FG-3154, a fully human neutralizing $\mathrm{mAb}$ recognizing CTGF, and a human control IgG were provided by FibroGen. CP was induced by i.p. injection of cerulein $(50 \mu \mathrm{g} /$ kg) 3 days per week for 7 weeks. During the final 2 weeks of cerulein injection, $40 \mathrm{mg} / \mathrm{kg} \mathrm{FG-3154}$ or control IgG was administered twice weekly by i.p. injection.

Human samples. Six pancreas samples of obstructive pancreatitis tissues and nonpancreatitis tissues were collected from patients with pancreatic body cancer who underwent distal pancreatectomy. In addition, 6 pancreas samples of CP tissues were collected from 3 patients with alcoholic CP, 2 patients with tumor-forming pancreatitis, and 1 patient with familial pancreatitis, all of whom underwent pancreaticoduodenectomy or distal pancreatectomy. Six samples of normal pancreatic tissues were collected from patients with pancreatic neuroendocrine tumors who underwent pancreaticoduodenectomy or distal pancreatectomy. All surgeries were performed at the Department of Gastroenterological Surgery, Osaka University Graduate School of Medicine, between February 2011 and April 2018.

Statistics. Statistical analyses were performed using Prism 9.0.1 software (GraphPad). Data are presented as the means \pm SDs. Twotailed Student's $t$ test was used to evaluate differences between 2 groups. One-way ANOVA with Tukey's post hoc test was used to compare differences among 3 or more groups. The survival data were analyzed using the log-rank test. A $P$ value less than 0.05 was considered significant.

Study approval. All animal experimental procedures were performed in accordance with the Osaka University guidelines for animal experiments and approved by the Ethics Committee of Osaka University Graduate School of Medicine. The use of resected human samples 
was approved by the Ethics Committee of Osaka University Graduate School of Medicine (protocol 17160), and written informed consent was received from all patients. The study design was consistent with the principles of the Declaration of Helsinki.

\section{Author contributions}

$\mathrm{T}$ Tamura, TK, RLJ, and $\mathrm{T}$ Takehara conceived the project and wrote, reviewed, and edited the manuscript. T Tamura, TK, and $\mathrm{T}$ Takehara collected and analyzed data. $\mathrm{T}$ Tamura performed most of the experiments, with contributions from TK, MS, HH, and $\mathrm{T}$ Takehara. TK and $\mathrm{T}$ Takehara acquired funding. $\mathrm{T}$ Takehara administered and supervised the project. KS, KM, TY, RY, RS, HA, HE, HY, MM, and T Tatsumi provided resources. The order of first authorship was determined by levels of contribution to manuscript writing.

\section{Acknowledgments}

This work was partially supported by a Grant-in-Aid for Scientific Research (to T Takehara) from the Ministry of Education, Culture, Sports, Science, and Technology, Japan. This work was supported by the Cancer Prevention Research Institute of Texas under grant RP180530 (to RLJ) and the Japan Agency for Medical Research and Development (AMED) under grant JP20fk0210074 (to TK). The authors thank FibroGen for providing the anti-CTGF neutralizing antibody FG-3154.

Address correspondence to: Tetsuo Takehara, Department of Gastroenterology and Hepatology, Osaka University Graduate School of Medicine, 2-2 Yamadaoka Suita, Osaka 565-0871, Japan. Phone: 81.6.6879.3621; Email: takehara@gh.med.osaka-u.ac.jp.
1. Kloppel G. Chronic pancreatitis, pseudotumors and other tumor-like lesions. Mod Pathol. 2007;20 (suppl 1):S113-S131.

2. Masamune A, et al. Variants that affect function of calcium channel TRPV6 are associated with early-onset chronic pancreatitis. Gastroenterology. 2020;158(6):1626-1641.

3. Lew D, et al. Chronic pancreatitis: current status and challenges for prevention and treatment. Dig Dis Sci. 2017;62(7):1702-1712.

4. Lowenfels AB, et al. Pancreatitis and the risk of pancreatic cancer. International Pancreatitis Study Group. NEnglJMed.1993;328(20):1433-1437.

5. Zhu L, et al. Acinar cells contribute to the molecular heterogeneity of pancreatic intraepithelial neoplasia. Am J Pathol. 2007;171(1):263-273.

6. Pinho AV, et al. Chronic pancreatitis: a path to pancreatic cancer. Cancer Lett. 2014;345(2):203-209.

7. Hill R, et al. PTEN loss accelerates KrasG12Dinduced pancreatic cancer development. Cancer Res. 2010;70(18):7114-7124.

8. Ying $\mathrm{H}$, et al. PTEN is a major tumor suppressor in pancreatic ductal adenocarcinoma and regulates an NF-кB-cytokine network. Cancer Discov. 2011;1(2):158-169.

9. Gruber R, et al. YAP1 and TAZ control pancreatic cancer initiation in mice by direct up-regulation of JAK-STAT3 signaling. Gastroenterology. 2016;151(3):526-539.

10. Hay N, Sonenberg N. Upstream and downstream of mTOR. Genes Dev. 2004;18(16):1926-1945.

11. Zhao B, et al. The Hippo pathway in organ size control, tissue regeneration and stem cell selfrenewal. Nat Cell Biol. 2011;13(8):877-883.

12. Ansari D, et al. The hippo signaling pathway in pancreatic cancer. Anticancer Res. 2019;39(7):3317-3321.

13. Wang L, et al. Expression profile and prognostic value of SAV1 in patients with pancreatic ductal adenocarcinoma [published online October 17, 2021]. Tumour Biol. https://doi.org/10.1007/ s13277-016-5457-4.

14. Wang L, et al. Protein salvador homolog 1 acts as a tumor suppressor and is modulated by hypermethylation in pancreatic ductal adenocarcinoma. Oncotarget. 2017;8(38):62953-62961.

15. Morvaridi S, et al. Role of YAP and TAZ in pancreatic ductal adenocarcinoma and in stellate cells associated with cancer and chronic pancreatitis. Sci Rep. 2015;5:16759.

16. Bellizzi AM, et al. The mTOR pathway is frequently activated in pancreatic ductal adenocarcinoma and chronic pancreatitis. Appl Immunohistochem Mol Morphol. 2010;18(5):442-447.

17. Xue J, et al. Alternatively activated macrophages promote pancreatic fibrosis in chronic pancreatitis. Nat Commun. 2015;6:7158.

18. Xu X, et al. Beta cells can be generated from endogenous progenitors in injured adult mouse pancreas. Cell. 2008;132(2):197-207.

19. Lau LF. Cell surface receptors for CCN proteins. J Cell Commun Signal. 2016;10(2):121-127.

20. Piccolo S, et al. The biology of YAP/TAZ: hippo signaling and beyond. Physiol Rev. 2014;94(4):1287-1312.

21. Matys V, et al. TRANSFAC and its module TRANSCompel: transcriptional gene regulation in eukaryotes. Nucleic Acids Res. 2006;34(suppl 1):D108-D110.

22. Dooher JE, et al. C/EBP $\alpha, \mathrm{C} / \mathrm{EBP} \alpha$ oncoproteins, or $\mathrm{C} / \mathrm{EBP} \beta$ preferentially bind $\mathrm{NF}-\kappa \mathrm{B}$ p 50 compared with $\mathrm{p} 65$, focusing therapeutic targeting on the C/EBP:p50 interaction. Mol Cancer Res. 2011;9(10):1395-1405.

23. Koschmieder S, et al. Dysregulation of the C/ EBPalpha differentiation pathway in human cancer. J Clin Oncol. 2009;27(4):619-628.

24. Wiwi CA, et al. Sexually dimorphic $\mathrm{P} 450$ gene expression in liver-specific hepatocyte nuclear factor 4alpha-deficient mice. Mol Endocrinol. 2004;18(8):1975-1987.

25. Alonso-Vale MI, et al. Adipocyte differentiation is inhibited by melatonin through the regulation of C/EBPbeta transcriptional activity. J Pineal Res. 2009;47(3):221-227.

26. Fu Y, et al. Scutellarin exerts protective effects against atherosclerosis in rats by regulating the Hippo-FOXO3A and PI3K/AKT signaling pathways. JCell Physiol. 2019;234(10):18131-18145.

27. Zhao Y, et al. PI3K positively regulates YAP and TAZ in mammary tumorigenesis through multiple signaling pathways. Mol Cancer Res. 2018;16(6):1046-1058.

28. Xu W, et al. PTEN lipid phosphatase inactivation links the hippo and PI3K/Akt pathways to induce gastric tumorigenesis. JExp Clin Cancer Res.
2018;37(1):198

29. Kodama T, et al. Molecular profiling of nonalcoholic fatty liver disease-associated hepatocellular carcinoma using SB transposon mutagenesis. Proc Natl Acad Sci US A. 2018;115(44):E10417-E10426.

30. Tumaneng K, et al. YAP mediates crosstalk between the Hippo and PI(3)K-TOR pathways by suppressing PTEN via miR-29. Nat Cell Biol. 2012;14(12):1322-1329.

31. Liu J, et al. Activation of the intrinsic fibroinflammatory program in adult pancreatic acinar cells triggered by Hippo signaling disruption. PLOS Biol. 2019;17(9):e3000418.

32. Rishi V, et al. CpG methylation of half-CRE sequences creates $\mathrm{C} / \mathrm{EBPalph}$ binding sites that activate some tissue-specific genes. Proc Natl Acad Sci U S A. 2010;107(47):20311-20316.

33. Jules J, et al. CCAAT/enhancer-binding protein $\alpha(\mathrm{C} / \mathrm{EBP} \alpha)$ is important for osteoclast differentiation and activity. J Biol Chem. 2016;291(31):16390-16403.

34. Flodby P, et al. Increased hepatic cell proliferation and lung abnormalities in mice deficient in CCAAT/enhancer binding protein alpha. J Biol Chem. 1996;271(40):24753-24760.

35. Kumagai T, et al. Epigenetic regulation and molecular characterization of C/EBPalpha in pancreatic cancer cells. Int J Cancer. 2009;124(4):827-833.

36. Zhang Z, et al. Activin a promotes myofibroblast differentiation of endometrial mesenchymal stem cells via STAT3-dependent Smad/CTGF pathway. Cell Commun Signal. 2019;17(1):45.

37. Dorn LE, et al. CTGF/CCN2 is an autocrine regulator of cardiac fibrosis. JMol Cell Cardiol. 2018;121:205-211.

38. Charrier A, Brigstock DR. Regulation of pancreatic function by connective tissue growth factor (CTGF, CCN2). Cytokine Growth Factor Rev. 2013;24(1):59-68.

39. Kodama $\mathrm{T}$, et al. Increases in $\mathrm{p} 53$ expression induce CTGF synthesis by mouse and human hepatocytes and result in liver fibrosis in mice. JClin Invest. 2011;121(8):3343-3356.

40. Makino Y, et al. CTGF mediates tumor-stroma interactions between hepatoma cells and hepatic stellate cells to accelerate HCC progression. 
Cancer Res. 2018;78(17):4902-4914.

41. Rachfal AW, Brigstock DR. Connective tissue growth factor (CTGF/CCN2) in hepatic fibrosis. Hepatol Res. 2003;26(1):1-9.

42. Gressner OA, Gressner AM. Connective tissue growth factor: a fibrogenic master switch in fibrotic liver diseases. Liver Int. 2008;28(8):1065-1079.

43. Charrier A, et al. Regulation of pancreatic inflammation by connective tissue growth factor (CTGF/CCN2). Immunology. 2014;141(4):564-576.

44. di Mola FF, et al. Connective tissue growth factor is a regulator for fibrosis in human chronic pancreatitis. Ann Surg. 1999;230(1):63-71.

45. Lu L, et al. Hippo signaling is a potent in vivo growth and tumor suppressor pathway in the mammalian liver. Proc Natl Acad Sci U S A. 2010;107(4):1437-1442.

46. Toda N, et al. Crucial role of mesangial cell- derived connective tissue growth factor in a mouse model of anti-glomerular basement membrane glomerulonephritis. Sci Rep. 2017;7:42114.

47. Kodama T, et al. Thrombocytopenia exacerbates cholestasis-induced liver fibrosis in mice. Gastroenterology. 2010;138(7):2487-2498.

48. Leal AS, et al. The Rho/MRTF pathway inhibitor CCG-222740 reduces stellate cell activation and modulates immune cell populations in Kras. Sci Rep. 2019;9(1):7072. 